\title{
Suggested merger of mental and neurological illnesses is premature
}

\author{
Sue Bailey president, Wendy Burn dean, Nick Craddock treasurer, Laurence Mynors-Wallis registrar, \\ Peter Tyrer editor
}

Royal College of Psychiatrists, London SW1X 8PG, UK

White and colleagues' suggestion that the distinction between mental and neurological illnesses should disappear is premature. ${ }^{1}$ It is perfectly reasonable to argue that mental illnesses are brain diseases - this was clearly stated more than 150 years ago ${ }^{2}$ - but this is not a reason to combine mental and behavioural disorders and diseases of the nervous system in one classification.

Although all mental events have brain correlates, we currently have no unequivocal brain biomarkers for most mental disorders, and classification relies, however imperfectly, on clinical signs and symptoms. Thus assessments and interventions differ from many of those in neurological practice. When White and colleagues suggest "the first priority should be to merge the mental and behavioural and neurology chapters of ICD-11 [international classification of diseases, 11th revision]" they may not be aware that each diagnosis in ICD-11 has a unique diagnostic number that can appear in more than one group in the classification while preserving its primary allocation for statistical purposes. Along with scientific validity, a central guiding principle of ICD-11 is clinical utility. Every good classification satisfies the needs of its users. With greater knowledge the systems may merge, but to do it now would not help professionals of either discipline.

Patients are more interested in the care they receive than in questions of nosology. Excellent clinical care depends on access to psychiatrists and neurologists who can liaise effectively but do not need to be yoked prematurely to an unwieldy classification system.

Competing interests: None declared.

1 White PD, Rickards H, Zeman AZJ. Time to end the distinction between mental and neurological illnesses. BMJ 2012;344:e3454. (24 May.)

Bucknill JC. The diagnosis of insanity. Br J Psychiatry 1856;2:229-45.

(c) BMJ Publishing Group Ltd 2012 